Providence College

DigitalCommons@Providence

$4-22-2020$

\title{
Surface mutations promote metal ion affinity in Haemophilus influenzae carbonic anhydrase
}

\author{
Ella Sheehan \\ Providence College
}

Follow this and additional works at: https://digitalcommons.providence.edu/chemistry_students

Part of the Biochemistry, Biophysics, and Structural Biology Commons

Sheehan, Ella, "Surface mutations promote metal ion affinity in Haemophilus influenzae carbonic anhydrase" (2020). Chemistry \& Biochemistry Student Scholarship. 6.

https://digitalcommons.providence.edu/chemistry_students/6

This Poster is brought to you for free and open access by the Chemistry \& Biochemistry at DigitalCommons@Providence. It has been accepted for inclusion in Chemistry \& Biochemistry Student Scholarship by an authorized administrator of DigitalCommons@Providence. For more information, please contact dps@providence.edu. 


\section{Surface mutations promote metal ion affinity in Haemophilus influenzae carbonic anhydrase}

Ella Sheehan and Kathleen Cornely, PhD

Department of Chemistry and Biochemistry, Providence College, Providence, RI 02918

\section{Introduction}

$\beta$-Carbonic Anhydrases ( $\beta$-CAs) are metalloenzymes that are essential to the growth of bacteria, making it a target for antibiotic research in the drug industry. For use in this research, the protein must be purified, and carbonic anhydrases native to a variety of bacterial species can be isolated via immobilized metal affinity chromatography (IMAC) due to the coordination of histidine tags on the protein's surface with the metal ions such as like Ni2+, promoting isolation of the bound protein from others in a sample. This IMAC approach was used to successfully enhance metal ion affinity with the Haemophilus influenzae carbonic anhydrase (HICA), by introducing histidine residues on the surface of a HICA protein via site-directed mutagenesis. The carbonic anhydrases isolated were eluted on a column using increasing concentrations of imidazole, which demonstrate the ion affinity on the surface of these metalloenzymes. An SDS-PAGE analysis was completed with all the isolated mutants, and this demonstrated successful overexpression.

\section{Background}

E. coli carbonic anhydrase (ECCA) have endogenous nickel affinity, and we used their structure to recreate the affinity in the HICA protein by completing a likely histidine cluster using site-directed mutagenesis. The HICA mutations resulted in mutant proteins that mimicked the endogenous affinity of ECCA to Ni-NTA, without the use of a random coil tag. This work paved the way for a number of mutations to be formed. Many questions remain unanswered however, namely, are there any limitations to how many histidine residues can be introduced and how might the mutations affect the kinetics of the carbonic anhydrase?
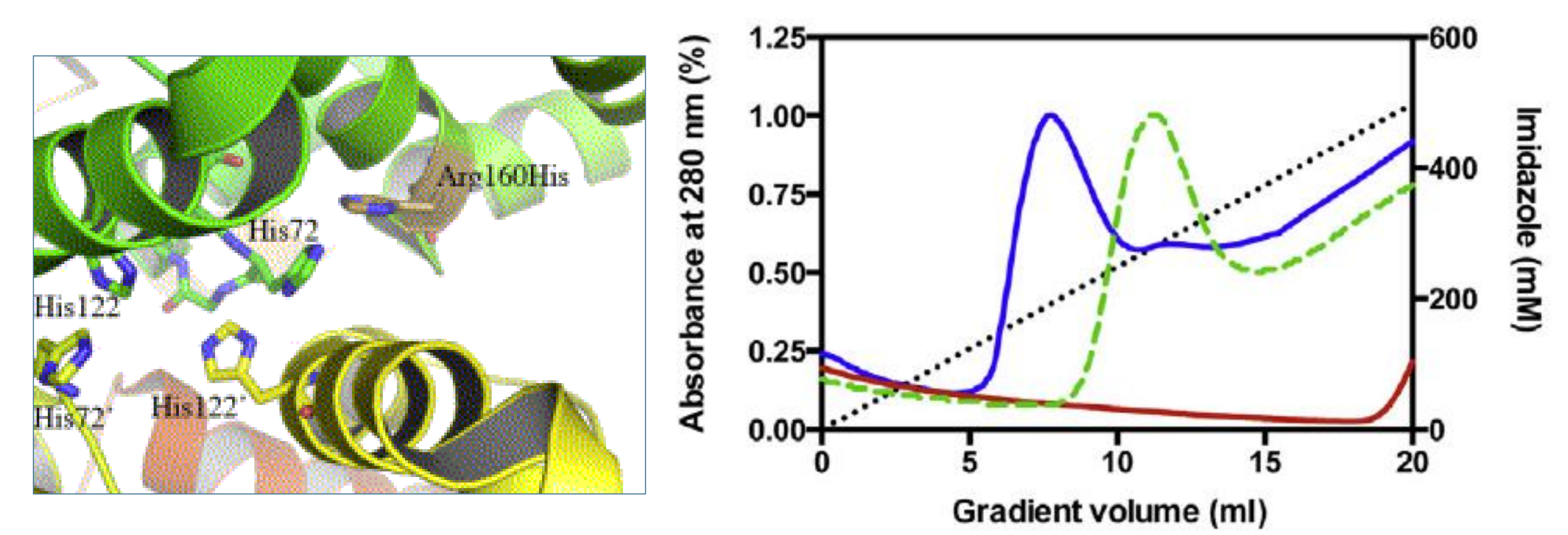

igure 1. Histidine tags. This is a sit Figure 2. HICA and ECCA elution profile. ECCA in which a cluster of histidine (green) has endogenous metal affinity due to the residues are positioned optimally to presence of surface-clustered His residues (Fig. 1) be able to bind to nickel, especially and elutes at $278 \mathrm{mM}$ imidazole. Wild-type HICA
considering that this interface (red) does not bind to a nickel column. R160H between monomers, one shown in HICA (blue) has a greater affinity for nickel than

green and the other shown in orange wild-type HICA and elutes from the column at 190 of the HICA tetramer are partially of the HICA tetram $\mathrm{mM}$ imidazole (1)

\section{Experimental Design}

PyMOL modeling software was used to design His mutant clusters varying in distance and number (2).

Plasmids expressing the mutant HICA gene were constructed using site-directed mutagenesis.

Mutant plasmids were introduced into an expression vector and the mutant HICA protein was overexpressed.

Cell lysate was loaded onto a nickel-NTA column and the concentration of imidazole required to elute the mutant protein was determined (3).

- Protein was quantitated by BCA assay and fractions were analyzed by SDS-PAGE.

The Cary50 UV-Vis Spectrophotometer was used to begin trials of a kinetic assay of each mutant HICA sample.

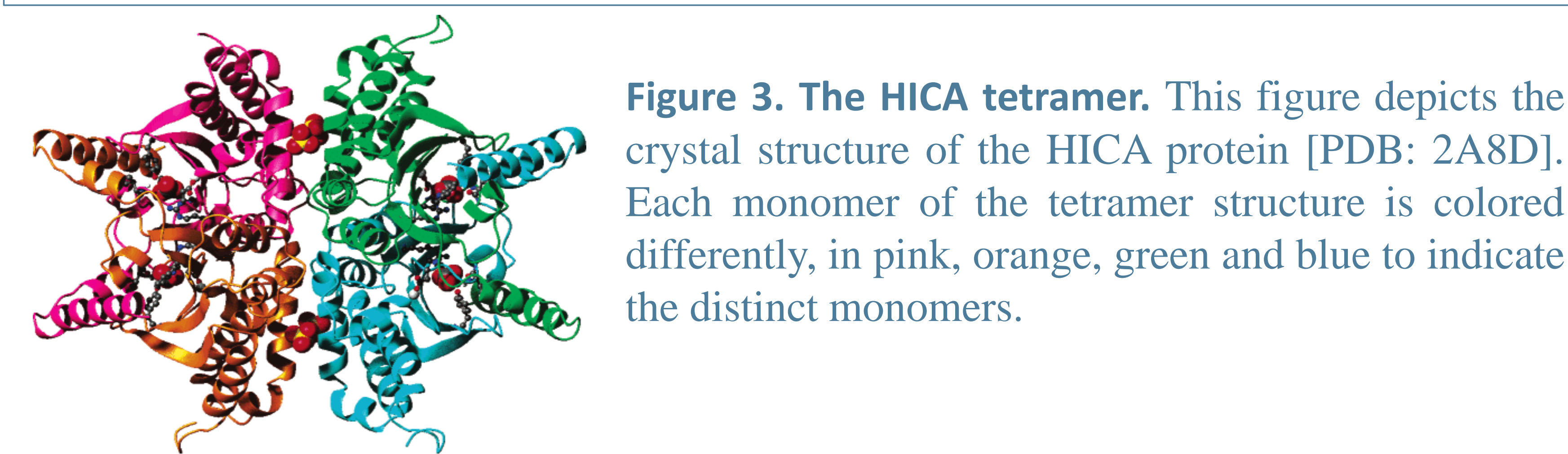

\section{Designed HICA mutants}

Once all mutants were designed, cloned and overexpressed, the cells were lysed, and this lysate was eluted on a Ni-NTA column with imidazole. The imidazole concentrations required of each mutant for elution demonstrates the strength of their ion affinity. If a greater imidazole concentration is observed, then that demonstrates enhanced ion affinity. The data in Table 1 below indicate the imidazole concentration required to elute each mutant.

Table 1: HICA mutants

\begin{tabular}{|c|c|}
\hline Mutation & [lmidazole] needed to elute (mM) \\
\hline S13H & 50 \\
\hline T34H & $50-100$ \\
\hline Y37H & $25-50$ \\
\hline E56+E59H & $150-200$ \\
\hline D125H & 25 \\
\hline I126H & 25 \\
\hline K129H & 25 \\
\hline L133H & 25 \\
\hline I126H+K129H & 100 \\
\hline K129+L133 & $50-100$ \\
\hline K136H & $50-100$ \\
\hline K136H+S138H+R142H & $100-150$ \\
\hline D144 & 25 \\
\hline I194 & 25 \\
\hline M194+T196 & $100-150$ \\
\hline T200H & $25-100$ \\
\hline M194+T196+T200H & 150 \\
\hline S204H & 50 \\
\hline S175H+T200H+S204H & 100 \\
\hline M194+T196+D216H+E218H & $100-200$ \\
\hline
\end{tabular}

\section{Results}

We designed a kinetic assay in which the HICA protein solution reacted with a substrate, 4-nitrophenyl acetate (4-NPA), in a PBS buffer, and as the 4-NPA was converted into the product, 4nitrophenol, there was a color change from a clear and colorless solution, to a clear yellow solution. To eliminate as many variables as possible, we controlled for temperature, enzyme concentration, and substrate concentration. Using the Cary50 UV-Vis Spectrophotometer, we quantified the rate of reaction in terms of the change in absorbance over time.

Our results for the kinetic assay are inconclusive at this time, however we were able to successfully confirm the presence of the overexpressed HICA within the cell lysate, assuring that the reaction that is occurring is catalyzed by the HICA enzyme. In figure 3, we show that thick bands, indicative of overexpression, formed from 11 mutant enzymes. The most distinct band in each of these samples is seen at a molecular weight of $19.0 \pm 0.3 \mathrm{kDa}$, which is consistent with monomers of the HICA tetramer.

Figure 3. SDS-PAGE gel. This gel contains 11 mutant HICA samples, and it identifies the presence of HICA in each lane at approximately $19.0 \mathrm{kDa}$ and identifies the overexpression.

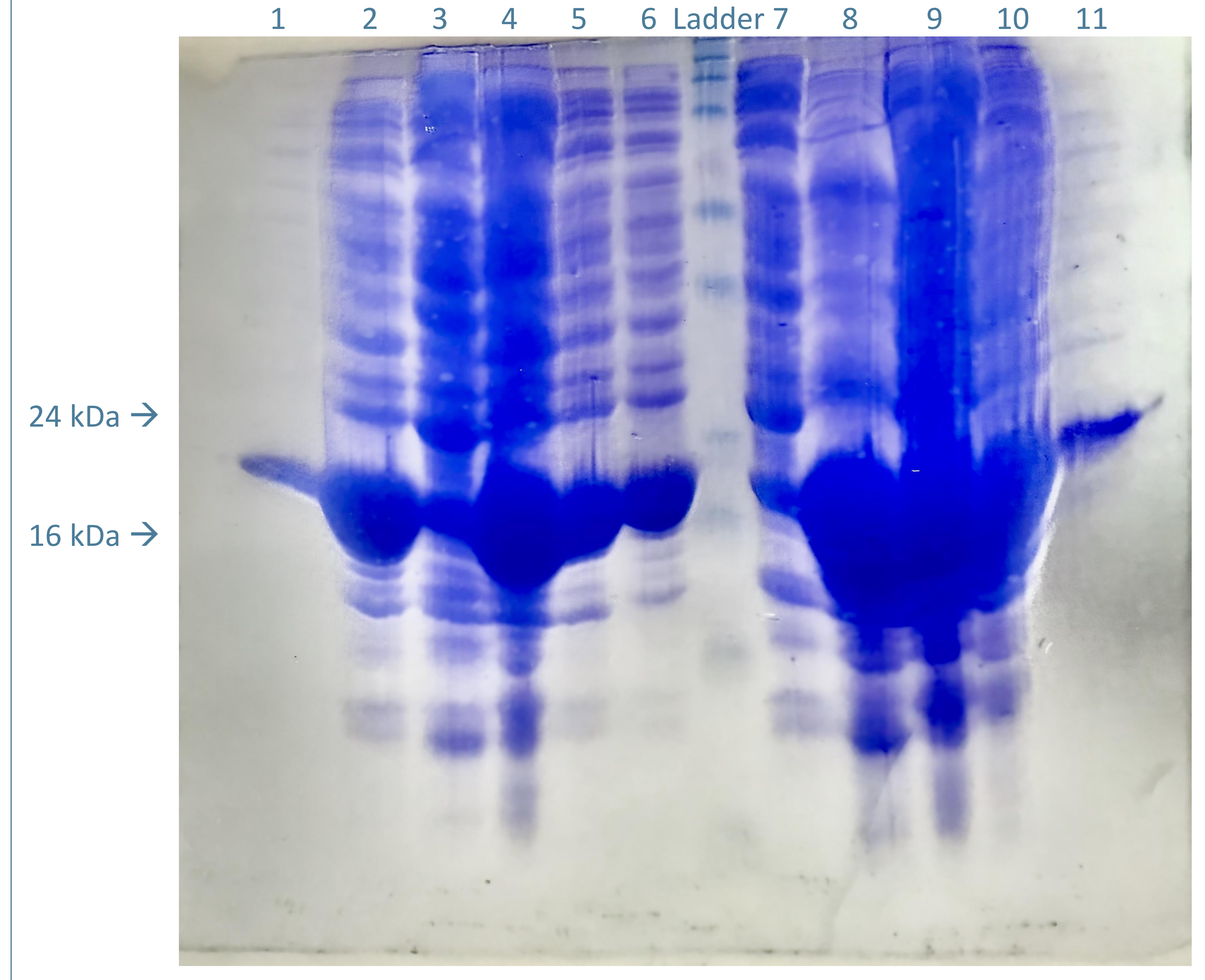

Further Research

The Cary50 UV-Vis Spectrophotometer will be used in future rials of a new kinetic assay protocol.

Based on the information we learned, it will be important that future assays are conducted in a neutral solvent, because any level of basicity can lead to base catalyzed hydrolysis of the substrate, which introduces error into the kinetic data.

Additionally, the Cornely lab may pursue the use of HICA's physiological substrate, carbon dioxide, for the assay to maximize the substrate specificity and ensure more accurate kinetic results.

\section{Thanks to the following:}

To Amy Goggin and the Center for Engaged Learning for organizing the Annual Celebration of Student Scholarship and Creativity.

To Katherine Hoffman of California Lutheran University for her generous supply of DH5A cells that contain the HICA plasmid to start each mutant.

To the faculty and staff in the Department of Chemistry and Biochemistry at Providence College.

To the CHM 310L students at Providence College and the Chem 443L students at Gonzaga University for piloting the research and designing the various mutants.

\section{References:}

. Hoffmann, K., Wood, K. M., Labrum, A., Lee, D. K., Bolinger, I. M., Konis, M. E., Blount, A. G., Prussia, G. A., Schroll, M. M. and Watson, J. M. (2014) Surface histidine mutations for the metal affinity purification of a $\beta$-carbonic anhydrase Anal.

DeLano, W. L. The PyMOL Molecular Graphics System (2002) DeLano Scientific, Palo Alto, CA USA

Block, H. Maertens, B., Spriestersbach, A. Brinker, N. Kubicek, J., Fabis, R., Labahn, J., and Schäfer, F. Chromatography (IMAC): A Review, Methods in Enzymology, 463, 439-473. 